\title{
Los valores y la investigación social
}

\author{
León Olivé \\ Director del Instituto de Investigaciones Filosóficas / UNAM
}

Ponencia presentada durante el IV Coloquio sobre Investigación Bibliotecologica llavado a cabo el 29, 30 y 31 de julio de 1985 en el CUIB

Los valores, sean propiamente cognoscitivos, éticos o estéticos, juegan un papel imprescindible en la investigación científica. Creo que hoy en día nadie disputaría la verdad de esta afirmación. Menos aún, entonces, se pondría en la tesis de que son imprescindibles en la investigación social. Debo precisar desde el principio que dejo abierta la posibilidad de hablar de investigación social no científica, la cual me parece que de modo aún más claro está sujeta a influencia de valores de diferente tipo.

Pero, ¿ cuál es el papel que juegan los valores, y cuál es su importancia?

La respuesta que se encuentra con mayor frecuencia, en mi opinión, reconoce que los valores condicionan (por lo menos, si no es que determinan) lo siguiente:

a) los fines en función de los cuales se investiga

b) la formulación de problemas

c) la selección de los problemas sobre los cuales de hecho se investiga

Esto permite plantearnos preguntas como; ¿hay siempre un interés instrumental en el conocimiento? Es decir, un interés en la predicción y el control. ¿Se hace siempre una investigación con el fin último de controlar el medio ambiente o ciertos procesos sociales? Un ejemplo de esto, pertinente en el contexto de esta plática, sería el interés en controlar el flujo de información y el acceso público a información especializada.

Una posición prototípica en este sentido aceptaría que los fines se fijan dentro de un marco conceptual amplio dentro del cual están incluidos los valores. 
Que los medios se eligen en función de esos fines. Por ejemplo, cuando un valor aceptado es el de la optimización de recursos, se buscarán los medios adecuados en ese sentido. Pero esta posición típicamente sostendría que una vez seleccionados los fines los cuales, repito, se admiten como determinados valorativamente los conocimientos que se produzcan en el proceso deben ser resultado de investigaciones científicas moralmente neutras, y que los contenidos mismos de esos conocimientos, cuando son auténticos, deben ser valorativamente neutrales y moralmente vacíos.

Por mi parte quiero compartir con ustedes algunas ideas en un sentido opuesto a las anteriores. Considero conveniente partir de la idea de que lo que debe considerarse como valorativamente neutral y moralmente vacía lo cual ya es en sí misma una postura valorativa es la naturaleza. Las sociedades, en cambio, no pueden serlo en virtud de lo que las hace sociedades de personas. Una idea que deseo enfatizar es que los conocimientos son elementos constitutivos de las sociedades de personas, y que tampoco pueden considerarse como valorativamente neutrales.

La tesis que deseo sostener y por lo menos indicar el sentido que tienen, así como la línea en la cual debe defenderse, es que los valores especialmente las tres tipos que ha mencionado: cognoscitivo, estéticos y morales son elementos que necesariamente deben estar en los entramados que a la vez son las condiciones de posibilidad de la producción de conocimiento, condicionan el contenido mismo de las creencias y conocimientos que resultan de los procesos de investigación basados en esos marcos. Ejemplo de cada uno de esos valores son claros: un valor ético en la investigación social puede plasmarse en un imperativo que prohiba al investigador realizar cierto tipo de experimentos con seres humanos. Un valor estético, se dice con frecuencia, puede ser el de apelar a un criterio como el de simplicidad, o elegancia para la aceptación de una teoría científica (dando por supuesto que no hay evidencia a favor o en contra que defina la aceptación). Por último, un claro valor cognoscitivo es el de la validez lógica. Ningún investigador actuando racionalmente, debería aceptar una teoría que contenga una contradicción lógica, o un argumento demostradamente inválido.

En la investigación social los valores juegan el papel que acabo de señalar para todo tipo de investigación con la pretensión de producir conocimiento. 
Pero en este caso su papel es doblemente importante porque, como también deseo sostener, los conocimientos son elementos constitutivos de la realidad social, es decir, de lo que se plantea como objeto de estudio para cualquier investigación de la sociedad.

Pero permítanme regresar a la cuestión que señale antes acerca de los fines de la ciencia o, para el caso, de la investigación sistemática. Comparemos las siguientes afirmaciones:

1) el propósito de la ciencia es la producción de conocimiento auténtico

2) el propósito de la ciencia es la intervención exitosa (manipulación y control) en el medio ambiente natural y en sociedad

3) el propósito de la ciencia es la expansión de la sabiduría social y personal

Quiero hacer notar que las tres afirmaciones no son lógicamente incompatibles, pero sí son independientes entre sí, y podrían servir de base para diferentes concepciones acerca de los fines de la actividad científica. Pero debe reconocerse que tal como están expresadas son evocativas y carecen de un contenido preciso. Para dotarlas de un contenido tal sería necesario analizar con detalle el significado de cada uno de los conceptos centrales, por ejemplo, conocimiento, sabiduría, verdad, racionalidad, explicación, predicción, (estos últimos sólo implícitos en las proposiciones mencionadas).

Por supuesto que todo esto sería excesivo para comentar y discutir en esta ocasión, pero la mención de estos problemas me parece un punto de partida adecuado para lo que quiero enfatizar en esta plática, y es que, si me permiten repetirlo, todo proceso de investigación presupone sistemas de valores y de normas, entre los que deben destacarse: los valores y normas cognoscitivas, metodológicas, y valores éticos y estéticos.

Antes de pasar a una explicación un poco más detallada, la cual en un principio será abstracta, quisiera ofrecer una ilustración de un valor particularmente, en mi opinión, en este contexto. Se trata del valor expresado a través 
de la idea de progreso en la adquisición y aplicación de conocimiento. Creo que es posible desarrollar un argumento en el sentido de que la aceptación de ese valor conduce necesariamente a la aceptación de la idea de que debe haber una irrestricta libertad en el manejo y flujo de información, en la comunicación entre quienes investigan, y que debe haber un acceso público a los resultados de las investigaciones. Me parece que debe ser de particular interés el problema de los mecanismos de almacenamientos y transmisión de información sobre los métodos y los resultados entre investigadores, y de los centros de investigación hacia el público más amplio.

Quisiera ahora, si me lo permiten, señalar de manera muy condensada cómo entiendo algunos de los conceptos básicos involucrados en cualquier discusión acerca de investigaciones con pretensiones de producir conocimiento, me refiero a las nociones de objetividad y de verdad.

En efecto, suele considerarse a la investigación científica como una actividad orientada hacia la verdad y uno de cuyos requisitos fundamentales es la objetividad. Y si bien podría aceptarse que ambos conceptos, objetividad y verdad llevan un valor en sí mismo, (un valor cognoscitivo), la idea más favorecida es que las actividades que conduzcan a la obtención de esos fines, así como los cuerpos de creencias y de conocimientos que se empleen como apoyos y como causas materiales, deben estar descontaminados de valores.

Lo que tratare de explicar a continuación son concepciones de la verdad, de la objetividad y del conocimiento, tales que nos permitan entender la relación intrínseca entre valores y conocimiento, lo cual puede servir de base para comprender la naturaleza y estructuras básicas de la investigación social.

Objetividad: la objetividad se refiere a la posibilidad de reconocimiento público, en una comunidad determinada, de que hay una situación de hecho. Dicho reconocimiento debería entenderse con respecto a cualquier realidad que tenga efectos en la sociedad en cuestión. El estatuto de real significa precisamente tener efectos en la sociedad de que se trate. Las creencias objetivas son, entonces, las que necesariamente tienen consecuencias en la sociedad en la cual se les sostiene. La objetividad de una creencia está en función de 
otras creencias disponibles, así como de los conocimientos y recursos materiales a los cuales tiene acceso una sociedad. Una creencia será objetiva en la medida en que no pueda ser rebatida dentro del marco conceptual del que dispone la comunidad en cuestión, y con la evidencia que los recursos teóricos y materiales a su disposición pueden ofrecer.

Por verdad, en cambio, sugiero entender aceptabilidad racional en condiciones epistémicas ideales. Esto significa, básicamente, que la verdad de una proposición no es relativa a los marcos conceptuales ni a la disposición de quienes interactúan y ponen en duda su validez. Si la discusión pudiera continuar por tanto tiempo como fuera necesario, pero además no hubiera ningún ejercicio de poder, y lo único que revaleciera fuera la fuerza del mejor argumento, entonces habría la posibilidad de que a partir de la interacción de los sujetos se transformaran los marcos conceptuales de los cuales partieron, y que dentro de un tercer marco conceptual así formado. Se llagara a reconocer la verdad de la proposición en disputa.

Una consecuencia interesante de lo anterior es que pueden haber creencias objetivas que no sean verdaderas, y viceversa.

Veamos primero qué significa que una proposición pueda no ser objetiva para una cierta comunidad, pero que sea verdadera. Por ejemplo, una proposición que exprese un saber genuinamente universal, digamos que una cierta estructura económica es de explotación y que por ello necesariamente induce, en las relaciones sociales, intereses particulares de grupo, los cuales no pueden satisfacerse plenamente dentro de tal estructura. Una conclusión que parecería extraerse de loa anterior sería la necesidad de transformar esa estructura para obtener otra que no genere esos conflictos, y por consiguiente, que resultara menos injusta. La cuestión es si tal proposición podría ser aceptada, estoes, si es aceptable, para todos los sujetos de la sociedad hipotética que nos planteamos. Tal como está construido el ejemplo, la respuesta es negativa. Eso significaría que tal proposición no expresa una creencia objetiva para tales sociedades.

Veamos ahora el sentido inverso. Si consideramos una jerarquía social, digamos J, entonces el sistema de creencias que tiene a su disposición es constitutivo de la realidad social misma. En dicho sistema se incluyen valores y normas. De ese modo, una proposición como "A es superior a B", donde A pertenece a un estrato de J, el cual según los valores, la estima y en general las creencias prevalecientes en $J$, es superior al estrato al cual pertenece B. En dicha comunidad es una creencia objetiva que A es superior a B. Desde otra 
perspectiva, sin embargo, tal afirmación puede ser rechazada con buenas razones. No sugiero con esto con esto que la proposición en cuestión sea verdadera en J, pero falsa para otras comunidades. El ejemplo quiere decir que es objetiva en J, pero falsa; aunque el meollo del asunto es que los miembros de J, mientras sigan siendo típico miembros socializados en J, no podrán rechazarla con buenas razones. En este caso, son precisamente inaccesibles para los miembros de J.

Lo que me interesa destacar ahora, en conexión con lo anterior, es la idea de un marco conceptual que necesariamente enmarca las actividades e interacciones sociales, incluyendo la investigación social. Un marco conceptual se puede comprender incluyendo por lo menos los siguientes principios:

a) una lógica que contiene por lo menos un principio débil de no contradicción, esto es, un principio que estipula que no toda proposición formulable en el lenguaje correspondiente puede ser verdadera, y que hay por lo menos un atributo que se aplica a instancias negativas, es decir, hay particulares a los cuales no se aplica:

b) un conjunto de conceptos intersubjetivos, es decir, conceptos que si se aplican a los objetos particulares que son accesibles a la experiencia de una persona, les confieren intersubjetividad; y, por consiguiente, un dominio de objetos particulares, a saber, las instancias de esos conceptos.

c) una categorización de los objetos intersubjetivos en clases maximales, es decir, clases que no pueden estar contenidas a la vez en otras;

d) para cada clase maximal:

- un principio constitutivo según el cual si un objeto es miembro de una clase maximal, entonces, por necesidad lógica, debe poseer un cierto atributo o un conjunto de atributos;

- en algunos casos, un principio de individuación según el cual el hecho de que un miembro de una clase maximal sea distinto de cualquier otro miembro implica lógicamente que posee un atributo o un conjunto de atributos;

e) en algunos casos, una clasificación de los objetos particulares y de los conceptos entre dependientes e independiente; 
f) en algunos casos, la admisibilidad de varias clases de conceptos auxiliares, es decir, conceptos que no son intersubjetivos, pero que sirven para la aplicación de éstos. (por ejemplo, idealización teórica o matemáticas) ${ }^{1}$;

g) un conjunto de valores de diversos tipos (éticos, estéticos y cognoscitivo),

h) un conjunto de norma, las cuales determinan las actitudes y orientan las prácticas

El esqueleto de lo que quiero sostener es entonces que todo sistema de acciones, particularmente la investigación social, y sobre todo los que como ella, están enmarcados por instituciones, presuponen un cierto marco categorial, y por ende grupos de valores y de normas que condicionan actitudes, prácticas, la selección de problemas, de creencias y conocimientos previos a la investigación, así como las metodologías e incluso el contenido mismo de los resultados.

Para precisar aún más las ideas, quisiera señalar los elementos más importantes que forman parte y que se presuponen en la actividad científica.

a) visiones del mundo, o ideologías totales (en el sentido de Mannheim),

b) matrices disciplinarias (en un sentido derivado del de Kuhn): incluye sobre todo valores, ejemplos paradigmáticos, y en general las creencias que definen un área como científica y la legitiman,

C) sistemas conceptuales presupuestos por las teorías, o familias de teorías, especialmente de tipo epistemológico. En gran medida estos presupuestos constituyen y caracterizan una parte de la realidad como objeto de estudio de una disciplina,

d) teorías,

e) sistemas conceptuales distinguidos dentro de teorías. Por ejemplo, modelos (que pueden ser explicativos), o bien leyes,

f) conceptos,

g) aplicaciones meramente conceptuales de una (s) teoría (s) o otra (s). Por ejemplo, la aplicación de una teoría matemática a una teoría econó- 
mica, con independencia de la última para predicciones, o para la decisión sobre políticas económicas

En segundo lugar pueden mencionarse las prácticas científicas, entendiendo por esto, por ejemplo, prácticas de observación, de experimentación, de discusión y transmisión de resultados, de evaluación, etc. Dentro de este concepto de prácticas deben incluirse los sistemas normativos y de creencias, de donde se derivan lineamientos para el establecimientos de áreas específicas de interés.

También dentro de las prácticas científicas hay que incluir técnicas de investigación específicas, es decir, procedimientos y técnicas que se usan para la definición de un área de problema y para delimitar el alcance y legitimidad de sus intentos de solución.

En tercer término cabe señalar a la instituciones a través de las cuales se desarrollan las actividades científicas y tecnológicas, y por medio de las cuales se aplican sus resultados. Asimismo, hay que mencionar a los papeles (roles), a través de los cuales se manifiestan efectivamente las instituciones.

En cuarto lugar, pueden analizarse las aplicaciones que no son meramente conceptuales. Estas pueden llevarse a efecto dentro de un contexto puramente científico, con propósito de evaluación de teorías, pongamos por caso; por ejemplo, cuando una teoría se aplica para un diseño experimental mediante el cual se trata de poner a prueba a otra teoría; o bien, las aplicaciones pueden hacerse hacia el exterior de contextos científicos, lo cual puede conducir a efectos, posiblemente cambios, en el entorno natural o social.

Quisiera dejar ahora este nivel tan abstracto y referirme a tres ejemplos provenientes de diferentes campos de investigación en los cuales puede apreciarse el condicionamiento de teorías, metodologías y aún de la constitución del objeto científico por los marcos presupuestos como los que he mencionado.

El primer ejemplo que deseo mencionar trata de una controversia radical que originó con el artículo de David Bóhm de 1952 sobre una interpretación de la teoría cuántica en términos de variables ocultas². En ese artículo, Bóhm impugno las pruebas de imposibilidad de que una teoría de variables ocultas fuese compatible con la teoría cuántica, en particular la prueba que John von Neumann había ofrecido en 1932 en su libro sobre los fundamentos matemáticos de la teoría cuántica3. Un interesante análisis de esta controversia se en- 
cuentra en un artículo de Trevor Pinch "¿Qué es lo que una prueba hace si no prueba? Un estudio de las condiciones sociales y de las divisiones metafisicas que condujeron a un fracaso en la comunicación entre David Bohm y John von Neumann en relación con la física cuántica" ${ }^{4}$. Como se sugiere en el mismo título, el autor desea enfatizar la falla de comunicación y desarrollar un análisis sociológico a tal efecto. En este caso, Bohm pugnaba para que se aceptara una interpretación no ortodoxa de la teoría cuántica descalificando la prueba ampliamente aceptada de von Neumann. A dicha tendencia se le resistía desde la posición institucionalizada de la élite de los físicos cuánticos.

Pinch analiza la falla de comunicaciónentre Bóhm y vo Neumann a dos niveles. Por un lado el que llama "el modo de articulación en el área de investigación". Esto es, cuando el objeto de disputa, en nuestro ejemplo la teoría cuántica, forma parte de la particular área de interés de los científicos involucrados en la controversia; su trabajo cotidiano de hecho, consiste en una exploración de dicho objeto. En los términos que aquí hemos manejado, diríamos que a través de su trabajo cotidiano los científicos pugnan por constituir al objeto y hacer que se consolide tal constitución a través del reconocimiento de la comunidad. Por otro lado, se encuentra "el modo de articulación de la historia oficial" que tiene lugar cuando se hace referencia al objeto de conocimiento en un contexto distinto al del área intermedia de interés. Por ejemplo en libros de texto, artículos de divulgación, o ensayo de historia de la ciencia.

La falla de comunicación entre los dos científicos del ejemplo, dentro del modo de articulación en el área de investigación, propone Pinch.

"puede explicarse en términos de sus diferentes técnicas de investigación. Von Neumann era un matemáticos comprometido a unificar y a hacer riguroso los diversos enfoques a la teoría cuántica mediante el uso de la técnica de investigación de la axiomatización. Bóhm era un físico teórico comprometido a desarrollar nuevas teorías considerando la base ontológica y epistemológica de los conceptos físicos ${ }^{5}$.

Estas diferencias en las técnicas de investigación que corresponden a importantes diferencias dentro de lo que al principio llamé prácticas científicas separaron no sólo a los dos grandes científicos mencionados, sino que puede 
generalizarse distinguiendo por un lado a grupos de físicos, y por otro a matemáticos y lógicos cuánticos.

La interpretación de Bóhm fue en general resistida por los físicos cuánticos quienes daban por sentada la prueba de imposibilidad de von Neumann, pero quienes, por las mismas diferencias en cuanto a los principios y técnicas de investigación, no conocían detalladamente y afondo dicha prueba. Al parecer, el artículo que logro reconocimiento y estableció la invalidez de la prueba de von Neumann fue el escrito pot J.S. Bell en 1964, el cual se quedo congelado por dos años antes de la publicarse en 1966 en el Reviews of Modern Physics. ${ }^{6}$. Así, Pinch afirma.

"...la estabilización que duró 34 años de la confusión acerca de la validez de la prueba de von Neumaan puede...explicarse por las diferentes técnicas de investigación de los físicos teóricos que construían nuevas teorías de variables ocultas, y los matemáticos y lógicos cuánticos que legislaban con sus pruebas que tales teorías eran imposibles. La razón por la cual tomó 34 años encontrar el error en la prueba es que no había un número suficiente de físicos que usaran la técnica de la axiomatización, por lo cual la mayroría de los físicos no podrían investigar directamente la prueba"

Lo anterior señala aspectos de la controversia a nivel del modo de articulación en el área de investigación. Otros factores de carácter claramente sociológico son propuestos por Pinch a nivel del modo de articulación de la historia oficial. Para empezar, la mayoría de los físicos que resistían a Bóhm aducían la prueba de von Neuman tal como se había articulado en la historia oficial, ya que como se mencionó antes, pocos la conocían directamente y a fondo. Por otra parte, se destaca la estructura de autoridad, la cual era amenazada por la interpretación heterodoxa de Bóhnm:

"Al defender una interpretación heterodoxa, Bóhm desafiaba la autoridad de la élite [de los físicos cuánticos] poniendo en cuestión la legitimidad de sus inversiones previa en la interpretación de la teoría cuántica. El modo de articulación de la historia oficial de la prueba de von Neumann puede verse entonces como un intento de mantener una particular estructura de autoridad" ${ }^{9}$.

Pinch hace referencia todavía a otro factor importante de destacar aquí que determinó también el curso de la controversia y por consiguiente el intento de 
construir un cambio y que resulta explicativo de por qué se la resistía a tal intento. Se trata de un sipuesto metafísico que se ha llamado aritmomorfismo.

Esencialmente, esto se refiere a la idea de que el conocimiento científico es fundamentalmente aritmético, en el sentido de que está formado por elementos discretos, que pueden distinguirse entre sí, y son posiblemente infinitos en número. Así, se sostiene que hay una amplia tendencia entre los científicos, y particularmente entre los físicos por considerar que los legítimos conceptos en su ciencia deben ser lógicamente discretos y aditivos, a diferencia de conceptos que enfatizan formas y cualidades.

Pinch sugiere entonces que la prueba de von Neumann se adhiera al ideal aritmético y por eso resulta incompatible con una perspectiva como la de Bóhm la cual involucra "fundamental el desarrollo de consideraciones cualitativas y conceptos dialécticos, los cuales podían expresarse matemáticamente pero no dentro del entramado de la axiomatización" ${ }^{10}$ Bóhm se adhería, por ejemplo, al principio de complementareidad.

Independientemente de la adecuación del análisis de Pinch, aun si burdamente reconstruye el proceso, señala efectivamente un periodo por lo menos de 12 años durante el cual se resistió a la propuesta de Bóhm, es decir, no se le reconocía y por consiguiente no quedaba constituido ningún cambio dentro de la comunidad de físicos cuántico, y tal resistencia se basaba en diversos factores provenientes de atribuciones institucionalizadas a través del modo de articulación de la historia oficial y de la autoridad de la élite de los físicos cuánticos.

Para el otro caso que deseo ejemplificar basta mencionar cualquier controversia entre escuelas de investigación que hayan logrado un mínimo de estabilización. Se trata de cambios ya constituidos y claramente reconocidos por cierta comunidad, la cual por supuesto practica su disciplina de acuerdo con los puestos y técnicas que defiende su escuela, pero cada una de esas comunidades está inmersa en una más amplia que se reconoce entre otras cosas precisamente por la competencia. 
En particular, deseo hacer referencia a la controversia entre las escuelas de J.M. Baldwin y de E.B. Titcheener, representante de la escuela de Lipzig, acerca de reacciones simples en psicología hacia finales del sigloxIX, tal como ha sido analizada por Gernot Bóhm en su artículo "Normas cognoscitivas, intereses del conocimiento y la constitución del objeto científico" ${ }^{11}$.

En ese trabajo se pone en claro que los grupos chocaron, se resistían entre sí, porque cada uno diseñaba y realizaba experimentos a la luz de diferentes grupos de reglas que regulaban, por un lado, la manera de enfocar empíricamente al objeto de estudio, y por otro el discurso científico acerca del mismo objeto. A la vez, los diferentes conjuntos de reglas venían condicionados desde la concepción de cada grupo acerca de lo que era la psicología. La escuela de Lipzig consideraba que toda la psicología empieza con la introspección. Para Baldwin no era así. Esto indujo grandes diferencias en lo que se buscaba en los experimentos, e incluso en la selección de sujetos experimentales. El resultado fue que en cada caso se constituyó un objeto de estudio distinto. Pero eso no fue aparente para los particulares en la controversia. Así, cada escuela había constituido efectivamente su objeto de estudio y logrado una cierta estabilidad, pero se resistían entre sí, sobre cuestiones que hasta entonces no rebasan el ámbito puramente científico, porque habían articulado diferentes intereses cognoscitivos.

Por último un ejemplo contemporáneo de un cambio ya constituido en un área científica pero que ha logrado sólo un reconocimiento limitado dentro de un subsistema de la comunidad científica, lo encontramos en el campo de la sociología con las escuelas que defienden y pugnan por generalizar el enfoque etnometodológico y la sociología de la vida cotidiana. Así, Jack Douglas, uno de los más enérgicos defensores de esa línea, en su ensayo de presentación al volumen Understanding Everyday Life afirma:

\footnotetext{
"hasta muy recientemente pocos sociólogos se dieron cuenta que la comprensión de la vida cotidiana debe ser el fundamento de toda la investigación y teoría sociológica; y todavía menos actuaron de acuerdo con este hecho crucial. Las razones para esto estaban basadas en la compleja red de supuestos de la empresa sociológica tradicional, los cuales a la vez estaban basados en los supuestos de la ciencia clásica y en las organizaciones oficiales de control en las sociedades occidentales" ${ }^{12}$.
} 
La gran cantidad de investigaciones desarrolladas en esa línea es suficiente para justificar la afirmación de que ha constituido un cambio dentro del ámbito de la sociología y se trata de una escuela con sus propios supuestos, normas, practicas de investigación, instituciones, etc. pero también es claro que sólo cubre una parte del campo de la sociología y que muchas otras escuelas le ofrecen resistencia.

Lo que he pretendido enfatizar es que los valores de diferentes tipo están intrínsecamente ligados a sistemas conceptuales que condicionan la selección de problemas, la constitución del objeto científico (o de investigación), la metodología que se aplica, las reglas que supone esa metodología, así como el contenido mismo de las creencias y conocimientos requeridos para el proceso de investigación.

Recurriendo a la terminología que introduje al principio, debemos entender que los valores incluyen en general categorías maximales, y que hay normas que justifican acciones sin tener a la vez otras normas previas que las justifiquen.

La justificación de las mismas debe darse en un proceso argumentativo el cual idealmente debe permitir la interacción de todos los sujetos involucrados y, más aún, debería permitir la libre discusión entre sujetos racionales cualesquiera.

Me parece que no hay un código de conducta existente para la investigación social, y lo que aquí he discutido explicaría el por qué. Creo en cambio que en la práctica de la investigación existen sistemas normativos que incluyen normas maximales

La única recomendación que desde la perspectiva de la filosofía me atrevería a hacer a quienes se proponen la realización efectiva de políticas de investigación y del desarrollo de investigaciones sustantivas, es que la adopción de metodología, el señalamiento de fines, la selección de estrategias y de problemas, todo, presupone y está condicionado por valores que conviene hacer explícitos para aceptarlos o rechazarlos de forma racional, es decir, a través de la discusión más amplia y libre posible. 
NOTAS

1. Hasta aquí he seguido la formulación de Korner 1984, p. 18.

2. D. Bohm, "A suggested interpretation of the quantum theory in terms of Hiddem Varaibles, I and I". En : Phisical Review 85, 1952, pp. 166 193.

3. J. Von Neumann, Mathematical foundatiions of quantum mechanics, Princeton University Press, 1955 (Primera edición alemana 1932).

4. T.J. Pinch, "What does a proof do if it does not prove? A study of the social conditions and metaphysical divisions leading to David Bóhm and John von Neumann Failing to communicate in quantum physics", en: E. Mendelson, P. Weingart y R. Whitley (eds.), The social Production of Scientific Knowledge, Reidel: Dordrecht, 1977.

5. op. Cit., p.205.

6. J.S.Bell, "On the problem of hidden variables in quantum mechanics". En: Reviews of Modern Physics 38, 1966, pp. $447-452$.

7. T.J. Pinch, op. cit., p.206.

8. Aquí el término "inversión se refiere a una idea de Bourdieu según la cual "se ve a los científicos como comprometidos en una competencia para obtener un capital social de reconocimiento, en la cual siguen estrategias de inversión particulares para asegurar una ganancia..."Pinch, op. cit.'p.178.

9. T.J. Pinch, op. cit. p.206.

10. Ibid., p. 205 . 
11. G. Bohme, "Normas cognoscitivas, del conocimiento y la constitución del objeto científico : un ejemplo del funcionamiento de las reglas de la experimentación", En : L. Olivé (ed) La explicación Social del Conocimiento, México: UNAM, 1985.

12. J.D. Douglas, Understanding everyday life. Londres : Routledge and Kegan Paul, 1971, p.3. 